\title{
Janus 异官能基笼型倍半硅氧烷的合成研究
}

\author{
彭 军*, $a, b$ 邢玉秀 $a$ 许 凯*,a \\ ( ${ }^{a}$ 中国科学院广州化学研究所 广东省电子有机聚合物材料重点实验室 广州 510650) \\ ( ${ }^{b}$ 广东工业大学轻工化工学院 广州 510006)
}

\begin{abstract}
摘要 设计合成确切结构的异官能基笼型倍半硅氧烷一直是国际性的研究热点问题. 用 “自下而上” 的方法，以半笼 型结构倍半硅氧烷为模板，成功地制备出具有 Janus 结构的四乙烯基四氨基苯基、四乙烯基四苯基异官能基笼型倍半硅 氧烷. 并且, 产物在一些极性溶剂(如四氢呋喃、氯仿及二甲基亚砜)中有较好的溶解性, 为其衍生化及应用提供了保证. 对所制备的异官能基笼型倍半硅氧烷进行了全面的表征分析，证明其具有 Janus 异官结构特征及完整的笼型结构. 紫外 可见及荧光发射光谱证实了倍半硅氧烷笼型结构的电子携带及传输作用，反过来证明其具有完整的笼型结构，同时也 为其在有机光电材料中应用提供可能. 提供了一类通用合成方法, 拓宽了倍半硅氧烷功能单体的种类, 并拓展了其应 用范围.
\end{abstract}

关键词 异官能基笼型倍半硅氧烷; Janus 纳米粒子; 功能单体

\section{A Study on Synthesis of Janus Hetero-functional Cubic Silsesquioxanes}

\author{
Peng, Jun ${ }^{*, a, b} \quad$ Xing, Yuxiu ${ }^{a} \quad$ Xu, Kai ${ }^{*, a}$ \\ ( ${ }^{a}$ Guangdong Provincial Key Laboratory of Organic Polymer Materials for Electronics, Guangzhou Institute of \\ Chemistry, Chinese Academy of Sciences, Guangzhou 510650) \\ ( ${ }^{b}$ School of Chemical Engineering and Light Industry, Guangdong University of Technology, Guangzhou 510006)
}

\begin{abstract}
Design and synthesis of well-defined hetero-functional cubic silsesquioxane remain as international research focuses and challenges. In this work, we demonstrated in detail the synthesis of two Janus hetero-functional cubic silsesquioxane. We applied similar "bottom-up" method to construct the aimed Janus HFCSQ, which was carried out by using cyclic tetrasiloxanes as synthetic platforms and followed by further hydrolysis and cage-closure. The obtained products exhibited good solubility in THF, $\mathrm{CHCl}_{3}$ and $\mathrm{DMSO}$, which embodied themselves potential use in further derivations and processing. The precise structure of these obtained Janus HFCSQ were fully characterized by FTIR, ${ }^{1} \mathrm{H}$ NMR, Maldi-tof MS, ${ }^{29} \mathrm{Si}$ NMR, WAXD and TGA. Red-shifts observed from Photoluminescence UV-vis analysis further confirmed extended conjugation of the silsesquioxane cages, which in turn proved the formation of completed cages. This work provided us a versatile novel method to prepare the resulting Janus HFCSQs, which can widely expand the species of silsesquioxane monomers and applications as well.
\end{abstract}

Keywords hetero-functional cubic silsesquioxane; Janus nanoparticle; functional monomer

近年来，在纳米材料与技术领域，利用“自上而下” 构建纳米结构材料研究取得了重要的进展, 使得在纳米 尺度构建杂化结构材料成为一种可能, 这为相关科技工 作者提供了新的挑战与机遇 ${ }^{[1]}$. 笼型倍半硅氧烷(Cubic Silsesquioxane, CSQ), 作为一种日渐重要的纳米结构构
筑单元，由于其自身优异的机械及热稳定性能，在多个 领域内得到应用，如杂化高分子 ${ }^{[2]}$ 、光学材料 ${ }^{[3]}$ 、生物医

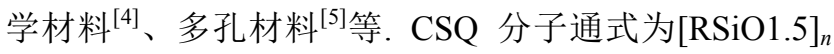
$(n=6,8,10,12)$, 是一类有机-无机分子内杂化笼型分 子结构，其内核为无机的 $\mathrm{Si}-\mathrm{O}-\mathrm{Si}$ 骨架，顶角带有有

* Corresponding authors. E-mail: pengjun3032@126.com, xk@gic.ac.cn

Received August 31, 2016; revised November 22, 2016; published online December 2, 2016.

Project supported by the National Natural Science Foundation of China (No. 21174162), the Province Natural Science Fund of Guangdong Province (No. 2016A030313162) and the Guangzhou Science and Technology Plan Projects (No. 201505051006333).

国家自然科学基金(No. 21174162)、广东省自然科学基金(No. 2016A030313162)及广州市科技计划(No. 201505051006333)资助项目。 
机官能基团 ${ }^{[6]}$. 目前, 研究最多的是高度对称结构的八 官能基立方笼型倍半硅氧烷, 然而, 倍半硅氧烷笼子的 衍生化反应受到顶角同官能基团的限制.

具有两种不同官能基团的笼型倍半硅氧烷异官能 基笼型倍半硅氧烷(Hetero-functional Cubic Silsesquioxane, HFCSQ)越来越引起研究者的兴趣. HFCSQ 能够拓 宽笼型倍半硅氧烷的应用, 如制备主链中含 CSQ 聚合 物、星型接枝聚合物及各向异性材料等等 ${ }^{[7]}$. 合成制备 具有准确结构的 HFCSQ 一直是本领域科研攻关的难题, 相关工作至今仍鲜有报道. 目前, 主要有五种方法制备 HFCSQ: (1)不同硅氧烷或氯硅烷单体直接共水解缩合 的方法 ${ }^{[8]}$; (2) 亲核试剂诱导重排的方法 ${ }^{[9]}$; (3)两类半笼 型倍半硅氧烷双盖帽的方法 ${ }^{[10]}$; (4)利用非完整笼型倍半 硅氧烷的顶点盖帽的方法 ${ }^{[11]}$; (5)全同官能基 CSQ 部分 官能化的方法 ${ }^{[12]}$. 然而, 上述制备方法的实例中, 很多 HFCSQ 都是只有一个活性的官能基团, 另一官能基团 大多数为烷基等惰性基团. 惰性集团无法满足进一步官 能衍生化的要求, 故此类 HFCSQ 不能称作真正意义上 的 HFCSQ. 不仅如此, 上述方法中所得的目标产物结 构不明确, 其中间产物也难以分离提纯. 不同硅氧烷或 氯硅烷单体共水解缩合的方法、重排法、部分官能化的 方法具有共同的产物复杂、分离难度大, 难以实现可控 合成的缺点, 因此, 我们在前期合成探索实验阶段积极 探索了一种利用非完整笼型倍半硅氧烷的顶点盖帽以 及两类半笼型倍半硅氧烷双盖帽的方法合成一类含有 双键及苯基的 HFCSQ 的方法. 非完整笼型倍半硅氧烷 的顶点盖帽的方法需要七聚苯基开笼型倍半硅氧烷前 驱体, 其合成途径一般有八苯基 CSQ 的开笼 ${ }^{[13]}$ 或苯基 三甲氧基硅烷的直接水解缩合 ${ }^{[14]}$. 我们发现, 采用七聚 苯基开笼型倍半硅氧烷前驱体, 通过乙烯基三氯硅烷 “顶角盖帽” 的方法能够得到乙烯基苯基 CSQ, 但是该 方法存在着七聚苯基开笼型倍半硅氧烷前驱体的结构 不确定、得率受限且有一定分离难度, 导致 HFCSQ 的 总体合成难度增大等缺点. 半笼型倍半硅氧烷双盖帽的 方法能够形成完整的笼型倍半硅氧烷结构, 但是存在结 构难以调控的缺点. 例如通过乙烯基半笼型倍半硅氧烷 与苯基半笼型倍半硅氧烷双盖帽的方法制备目标乙烯 基苯基 $\mathrm{HFCSQ}$, 结果表明其主要产物却为苯基 CSQ. 这主要是由于苯基 CSQ 的溶解性最差, 使得反应中的 溶解平衡被打破, 从而生成更多的苯基 $\mathrm{CSQ}^{[15]}$.

上述两种方法有一个共同点, 即从纳米结构单元而 非传统的分子单体出发构建目标 HFCSQ. 从分子设计 角度看, 半笼型倍半硅氧烷作为一种纳米模板单元, 可 成为有趣的合成完整笼型倍半硅氧烷的前体材料 ${ }^{[16]}$. 基于此, 我们提出了一种基于半笼型倍半硅氧烷结构
“自下而上” “生长” 合成目标 HFCSQ 的新方法. 本章 中, 我们将合成两类 HFCSQ, 即四乙烯基四氨基苯基 HFCSQ (TVTA-HFCSQ) 和四乙烯基四苯基 HFCSQ (TVTP-HFCSQ). 以相应的氨基苯基半笼型硅醇钠盐及 苯基半笼型硅醇钠盐为生长模板, 乙烯基三氯硅烷在四 甲基氢氧化铵( TMAH)/三乙胺 $\left(\mathrm{Et}_{3} \mathrm{~N}\right)$ 催化下水解链接生 长及成笼, 从而得到完整的具有双面结构的异官能基笼 型倍半硅氧烷. TVTA-HFCSQ 和 TVTP-HFCSQ 在四氢 呋喃、氯仿、二甲基亚砜等溶剂中展现了良好的溶解性, 且产物通过傅里叶红外光谱、核磁共振氢谱、飞行时间 质谱、硅谱、广角 X 射线衍射、热重分析等多种手段进 行表征, 证明其具有明确的结构.

\section{1 结果与讨论}

\section{1 前期研究探索}

1.1.1 七聚苯基开笼型三硅醇 $\left[\mathrm{Ph}-\mathrm{T}_{7}-(\mathrm{OH})_{3}\right]$ 及七聚 苯基开笼型三硅醇钠盐 $\left[\mathrm{Ph}-\mathrm{T}_{7}-(\mathrm{ONa})_{3}\right]$ 的制备

主要研究了利用非完整笼型倍半硅氧烷的顶点盖 帽的方法. 研究发现, 顶角盖帽的方法关键在于非完整 笼型倍半硅氧烷的可控制备. 为宏量制备 $\mathrm{Ph}-\mathrm{T}_{7}-(\mathrm{OH})_{3}$, 采用了两种合成路线: 一种是八苯基笼型倍半硅氧烷 (OPS)的可控开笼, 另一种是苯基三甲氧基硅烷直接水 解缩合的方法. 图 1 为不同催化剂(氢氧化钠、TMAH、 $\mathrm{Et}_{3} \mathrm{~N}$ 、邻磺酰苯甲酰亚胺及 $\mathrm{TBAF}$ ) 催化下 OPS 开笼反 应初产物的 ${ }^{1} \mathrm{H}$ NMR 数据. 从图 1 可以看出, 几类催化 剂开笼产物主要的峰位移保持一致, 在 $\delta 7 \sim 8$ 处有明显 的苯环质子氢的共振峰, 硅羟基的质子峰位移在 $\delta 0 \sim 2$ 间. 从核磁氢谱积分面积看, 积分面积比与 $\mathrm{Ph}-\mathrm{T}_{7}-(\mathrm{OH})_{3}$ 理论的苯环质子氢与硅羟基质子氢的个数比 $35: 3$ 不能 符合, 表明其产物结构并不只有目标的 $\mathrm{Ph}-\mathrm{T}_{7}-(\mathrm{OH})_{3}$, 为 不同程度开笼产物的混合体系. 虽然, 后续的薄层柱层 析的分离方法可以实现 $\mathrm{Ph}-\mathrm{T}_{7}-(\mathrm{OH})_{3}$ 的分离提纯, 但是 操作复杂、周期长、得率较低这些问题将限制了进一步 的盖帽. 进而, 我们尝试了苯基三甲氧基硅烷直接水解 缩合的方法制备 $\mathrm{Ph}-\mathrm{T}_{7}-(\mathrm{ONa})_{3}$. 从图 2 可以看出, $\mathrm{Ph}-\mathrm{T}_{7^{-}}$ $(\mathrm{ONa})_{3}$ 初产物的核磁谱图中, 主要有较强的苯环质子氢 共振峰以及疑似硅羟基的共振峰. 可以看出, 除了目标 的 $\mathrm{Ph}-\mathrm{T}_{7}-(\mathrm{ONa})_{3}$ 之外还存在一些副产物, 进一步提纯可 得到 $\mathrm{Ph}-\mathrm{T}_{7}-(\mathrm{ONa})_{3}$. 这两种方法都能得到七苯基非完整 笼型产物, 为后续的顶角盖帽法提供前体材料, 缺点在 于产物分离复杂且得率不高.

\subsection{2 半笼倍半硅氧烷双盖帽的方法制备 TVTP- HFCSQ 探索实验}

探究了四乙烯基环硅氧烷四硅醇(TVS)的合成与四 苯基环硅氧烷四硅醇(TPS)双盖帽的方法制备具有两面 


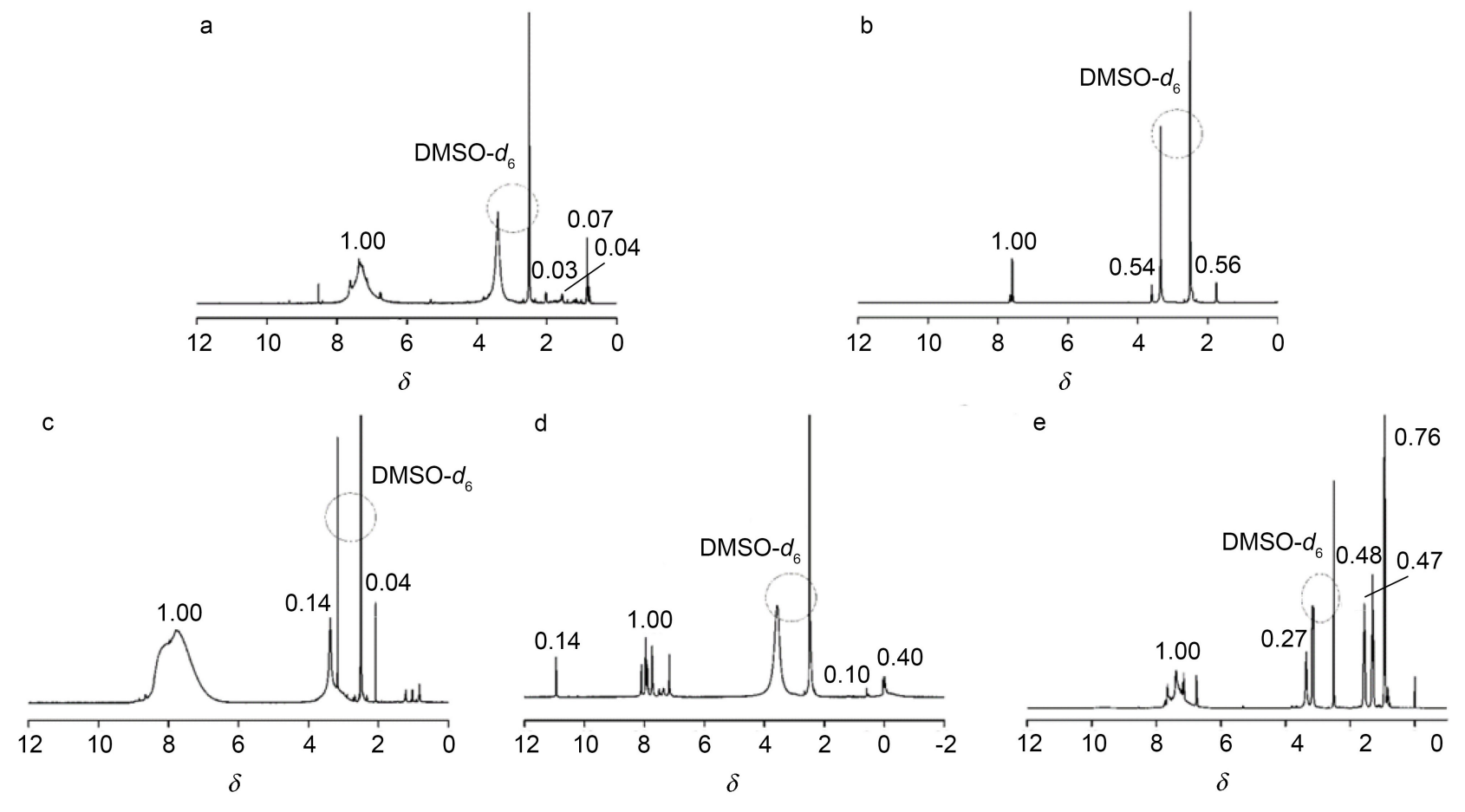

图 1 不同催化剂下 $\mathrm{OPS}$ 开笼反应得到 $\mathrm{Ph}_{-} \mathrm{T}_{7}-(\mathrm{OH})_{3}$ 的核磁谱图

Figure $1{ }^{1} \mathrm{H}$ NMR spectra of the obtained $\mathrm{Ph}-\mathrm{T}_{7}-(\mathrm{OH})_{3}$ raw products through cage-opening of OPS under various catalysts (a) $\mathrm{NaOH},(b) \mathrm{TMAH}$, (c) $\mathrm{Et}_{3} \mathrm{~N}$, (d) benzosulfimide, (e) $\mathrm{TBAF}$

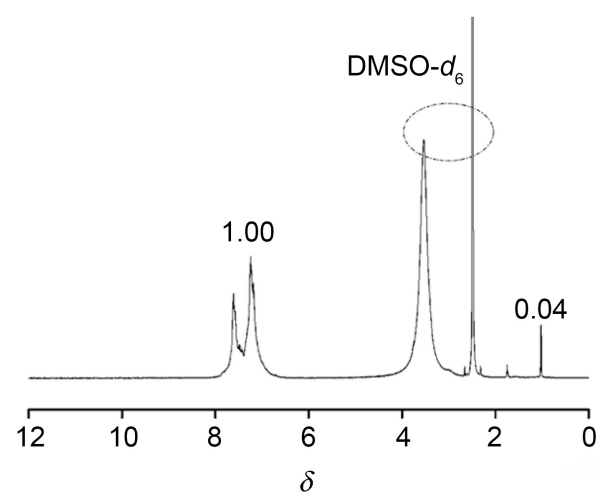

图 2 苯基三甲氧基硅烷直接水解缩合制备 $\mathrm{Ph}-\mathrm{T}_{7}-(\mathrm{ONa})_{3}$ 初产 物的核磁氢谱图

Figure $2{ }^{1} \mathrm{H}$ NMR spectrum of the prepared $\mathrm{Ph}-\mathrm{T}_{7}-(\mathrm{ONa})_{3}$ raw product by hydrolysis and condensation of phenyltrimethoxysilane

官能基团各异的 TVTP-HFCSQ. 如图 3 所示, 产物的核 磁氢谱中, 只有 $\delta 7.0 \sim 8.0$ 处有明显的苯环质子氢共振 峰, 乙烯基质子氢在 $\delta 6.0$ 附近并未观察到, 由此可见, 双盖帽的方法得到的产物可能为八苯基笼型倍半硅氧 烷. 可能是由于笼型产物的溶解性决定了其生成的速率 及结果. OPS 的溶解性较差, 因此双盖帽时, 更多的 TPS 自身缩合形成了 OPS. 双盖帽法作为一种潜在的制备双 面结构 HFCSQ 的方法仍有一些问题亟待克服.

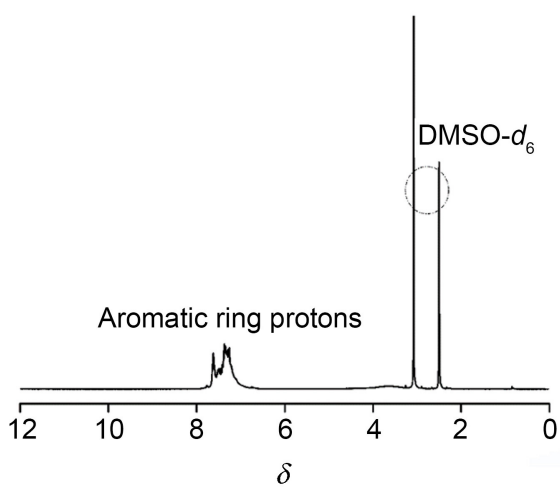

图 3 TVS 与 TPS 双盖帽反应制备 TVTP-HFCSQ Figure $3{ }^{1} \mathrm{H}$ NMR spectrum of the main product obtained from cross-condensation of TVS and TPS

\subsection{TVTA-HFCSQ 及 TVTP-HFCSQ 的设计合成}

TVTA-HFCSQ 及 TVTP-HFCSQ 的分子结构如图 4 所示. TVTA-HFCSQ 与 TVTP-HFCSQ 均具有无机 $\mathrm{Si}-$ $\mathrm{O}$ - $\mathrm{Si}$ 骨架结构, 顶角基团分别有氨基苯基、乙烯基及 苯基、乙烯基，是一类典型的 Janus 纳米粒子.

1.2.1 TVTA-HFCSQ 及 TVTP-HFCSQ 的完整笼型结 构及异官能基 Janus 结构特征

TVTA-HFCSQ 及 TVTP-HFCSQ 的合成路线如 Scheme 1 所示. 利用氢氧化钠催化苯基三甲氧基硅烷水 

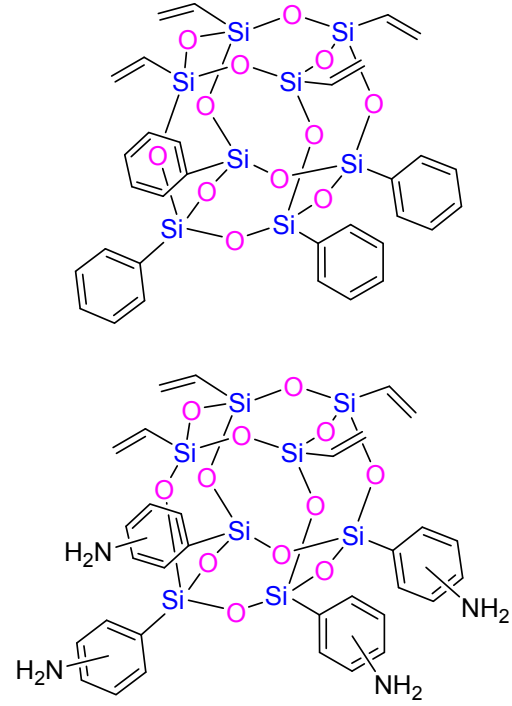

图 4 四乙烯基四苯基及四乙烯基四氨基苯基异官能基笼型 倍半硅氧烷(TVTA-HFCSQ 及 TVTP-HFCSQ)的结构式

Figure 4 Chemical structures of TVTA-HFCSQ and TVTPHFCSQ

解的方法, 成功地制备出四苯基环硅氧烷钠盐(TPSS). 其表征结果与文献报道符合 ${ }^{[17]}$. FT-IR (KBr) $v$ : 1583 与 $1460 \mathrm{~cm}^{-1}$ (苯环的骨架震动), $1116 \mathrm{~cm}^{-1}(\mathrm{~s}, \mathrm{Si}-\mathrm{O}-\mathrm{Si})$; ${ }^{1} \mathrm{H}$ NMR (DMSO- $\left.d_{6}\right) \delta: 6.0 \sim 7.8(\mathrm{~m}, 1 \mathrm{H})$, 核磁图谱除此 峰和溶剂峰之外无其他共振峰. TPSS 经过发烟硝酸硝 化反应得到四硝基苯基环硅氧烷钠盐. 进一步在水合肼 还原为四氨基苯基环硅氧烷钠盐(TASS). TASS 的表征 结果: FTIR (KBr) v: 1578 与 $1448 \mathrm{~cm}^{-1}$ (苯环的骨架震动
峰), 3369, $3220(\mathrm{w}, \mathrm{N}-\mathrm{H}), 1119 \mathrm{~cm}^{-1}$ (s, Si-O-Si); ${ }^{1} \mathrm{H}$ NMR $\left(\right.$ DMSO- $\left.d_{6}\right) \delta: 6.9 \sim 8.1(\mathrm{~d}, 2.0 \mathrm{H}), 5.3 \sim 3.7(\mathrm{~d}$, $1.0 \mathrm{H})$. 下一步通过乙烯基三氯硅烷的模板生长法及缩 合反应制备目标 TVTA-HFCSQ 和 TVTP-HFCSQ. $\mathrm{TMAH}$ 是一类很好的用于催化烷氧基硅烷单体水解的 催化剂, 且 $\mathrm{Et}_{3} \mathrm{~N}$ 催化条件下缩合反应更倾向于形成笼 型结构 ${ }^{[18]}$. 因此, 我们采取 $\mathrm{TMAH} / \mathrm{Et}_{3} \mathrm{~N}$ 复配催化体系 进行第二步的二甲氧基衍生物闭笼. TVTA-HFCSQ 和 TVTP-HFCSQ 的得率分别为 $37.4 \%$ 及 $40.1 \%$. TVTAHFCSQ 和 TVTP-HFCSQ 在常见有机溶剂如 $\mathrm{CHCl}_{3}$ 、 DMSQ 中有较好的溶解度, 其结构通过 FT-IR、 ${ }^{1} \mathrm{H}$ NMR、 ${ }^{29} \mathrm{Si}$ NMR、Maldi-tof MS 手段进行了表征, 结晶 性及热稳定性能分别由 WAXD 及 TGA 分析测得.

TVTA-HFCSQ 和 TVTP-HFCSQ 的两种活性官能基 团及内核的完整 $\mathrm{Si}-\mathrm{O}-\mathrm{Si}$ 骨架可以通过 FT-IR 光谱数 据初步确定. 从图 5 可以看到, TVTA-HFCSQ 和 TVTPHFCSQ 两者的红外谱图中, 分别在 $1117,1141 \mathrm{~cm}^{-1}$ 处 有强的 $\mathrm{Si}-\mathrm{O}-\mathrm{Si}$ 伸缩振动吸收峰, 表明了内核 $\mathrm{Si}-$ $\mathrm{O}-\mathrm{Si}$ 骨架的存在, 结合其在 $3600 \mathrm{~cm}^{-1}$ 附近没有 $\mathrm{Si}-$ $\mathrm{OH}$ 吸收峰, 可以初步判断其具有完整的笼型结构. 两 者谱图中同样存在 $3000 \mathrm{~cm}^{-1}$ 处附近的 $\mathrm{C}-\mathrm{H}$ 伸缩振动 峰、1432、1610 $\mathrm{cm}^{-1}$ 处明显的苯环骨架振动峰、 1625 $\mathrm{cm}^{-1}$ 处附近 $\mathrm{C}=\mathrm{C}$ 的特征振动吸收峰, 由此可见, TVTA-HFCSQ 和 TVTP-HFCSQ 两者化学结构上都拥有 苯环以及双键基团. TVTA-HFCSQ 的红外谱图在 3352 $\mathrm{cm}^{-1}$ 处有明显的 $\mathrm{N}-\mathrm{H}$ 振动吸收峰, 硝基的特征峰消<smiles>CO[Si](OC)(OC)c1ccccc1</smiles>

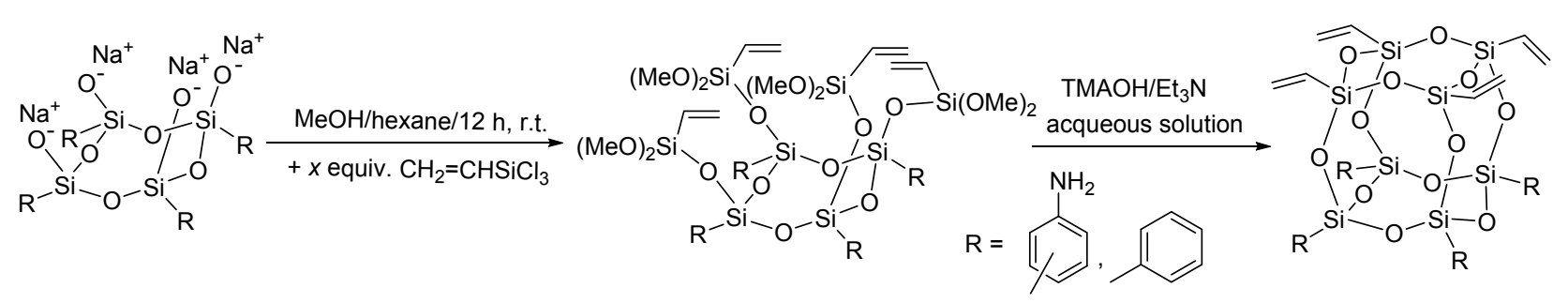

图式 1 TVTA-HFCSQ 及 TVTP-HFCSQ 的合成路线

Scheme 1 Proposed synthesis pathways for TVTA-HFCSQ and TVTP-HFCSQ 
失，表明硝基完全被还原.上述结果分析初步表明了 TVTA-HFCSQ 和 TVTP-HFCSQ 具有完整的笼型结构且 分别含有氨基苯基与双键以及苯环与双键两种活性官 能基团.

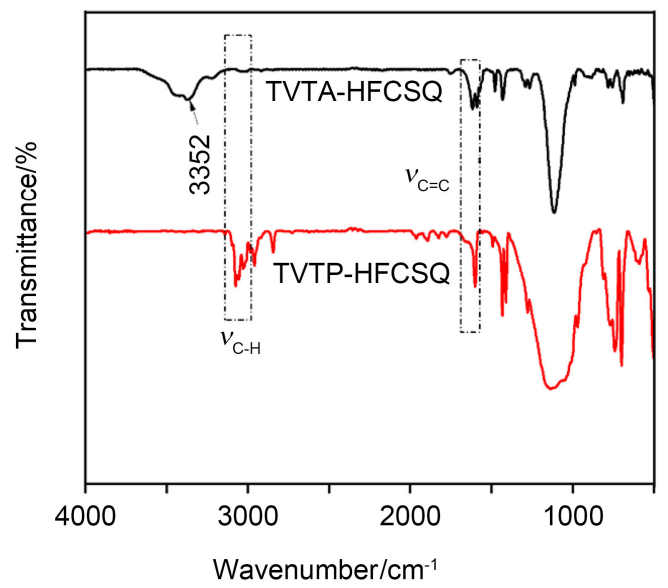

图 5 TVTA-HFCSQ 及 TVTP-HFCSQ 的红外谱图 Figure 5 FT-IR spectra of TVTA-HFCSQ and TVTP-HFCSQ

TVTA-HFCSQ 和 TVTP-HFCSQ 两者的核磁共振氢 谱数据进一步证实上述 FT-IR 分析的结果, 同时提供了 两种活性官能基团比率的数据. 如图 6 所示, TVTAHFCSQ 的核磁共振氢谱图中, 可以看到, 在 $\delta 7.28 \sim$ 7.81 处有较强的苯环质子氢产生的多重共振峰, $\delta$ 5.76 6.12 处为乙烯基氢原子产生的共振峰, $\delta$ 3.36 3.68 处为氨基氢原子产生共振峰, 三者的峰积分面积之 比为 $2.12: 1.56: 1$, 接近理论值 $4: 3: 2$, 说明 TVTA-HFCSQ 分子中氨基苯基与双键基团的个数相同, 为 Janus 纳米粒子. TVTP-HFCSQ 的核磁共振氢谱中, $\delta$ $7.18 \sim 7.75$ 为苯环质子氢的共振峰, $\delta 5.74 \sim 6.15$ 为乙烯 基氢原子共振峰, 两者的峰积分面积比为 $1.64: 1$, 接 近理论值 $5: 3$, 表明 TVTP-HFCSQ 分子中苯环与双键 个数相同, 结合制备方法的特点, 我们可推测其为具有 双面结构的 Janus 纳米粒子. 两者的核磁共振氢谱图中 没有出现 $\mathrm{Si}-\mathrm{OH}$ 及 $\mathrm{Si}-\mathrm{OCH}_{3}$ 氢原子的共振峰, 也进一 步表明了闭笼过程的完全进行, 形成了目标 Janus TVTA-HFCSQ 和 TVTP-HFCSQ 结构.

图 7 为 TVTA-HFCSQ 及 TVTP-HFCSQ 的核磁共振 硅谱图. 首先, 可以看到两者的硅谱图中都存在两种不 同环境硅原子的共振峰. 在 TVTA-HFCSQ 及 TVTPHFCSQ 硅谱图中, 分别在 $\delta-82.3,-77.3$ 处及 $\delta-$ $79.3,-76.2$ 产生了两组共振峰. $\delta-82.3$ 及一 79.3 处共 振峰被归属为与乙烯基基团链接的硅原子, 其位移的较 小变化与分子所处总体环境有关 ${ }^{[19]} ; \delta-77.3$, 及一 76.2 处共振峰分别归属为与氨基苯基及苯基键接的硅 原子 ${ }^{[20]}$. 其结果表明 TVTA-HFCSQ 及 TVTP-HFCSQ 均

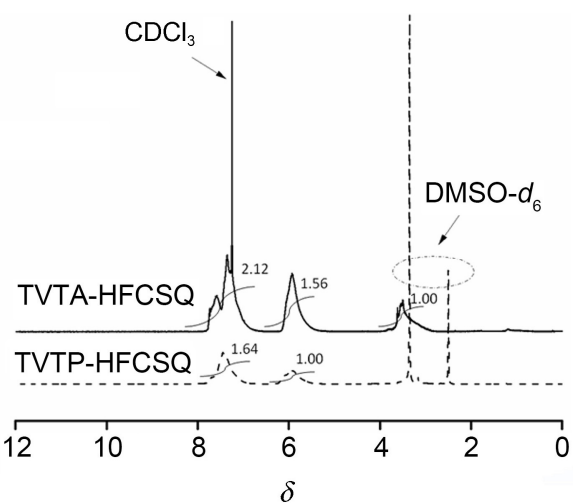

图 6 TVTA-HFCSQ 及 TVTP-HFCSQ 的 ${ }^{1} \mathrm{H}$ NMR 谱图 Figure $6{ }^{1} \mathrm{H}$ NMR spectra of TVTA-HFCSQ and TVTPHFCSQ

具有两种硅原子环境, 可作为 Janus 结构的佐证.
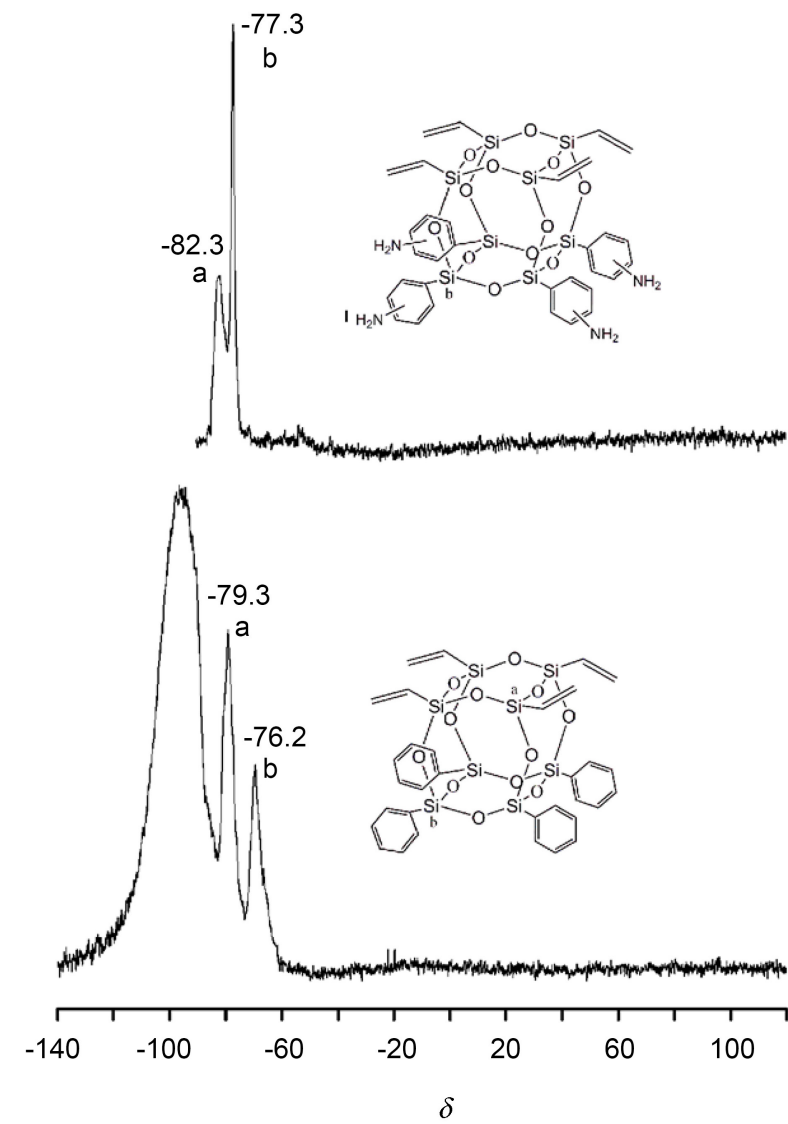

图 7 TVTA-HFCSQ 及 TVTP-HFCSQ 的 ${ }^{29} \mathrm{Si} \mathrm{NMR}$ 谱图 Figure 7 Identification of TVTA-HFCSQ and TVTP-HFCSQ by ${ }^{29} \mathrm{Si} \mathrm{NMR}$

图 8 是 TVTA-HFCSQ 及 TVTP-HFCSQ 的 Maldi-tof 质谱图. TVTA-HFCSQ 及 TVTP-HFCSQ 的 Maldi-tof 质 谱分别在 $m / z 918$ 及 856 处有较强的分子缔合峰，且没 有其他的分子峰，这说明产物具有较高的纯度. 通过右 上角局部放大的谱图可以看到，主峰分别在 $m / z 918$ 及 
856 处. 由于测试过程中会加入适量的钠盐辅助分子激 发, 所以测试结果为真实分子量加上 23, TVTA- HFCSQ 及 TVTP-HFCSQ 理论分子量为 892 及 832 , 加上 23 为 915 及 855 , 与测试值 918 及 856 很吻合. 综合 FT-IR、 ${ }^{1} \mathrm{H}$ NMR、 ${ }^{29} \mathrm{Si}$ NMR 及 Maldi-tof 质谱数据分析, 可知目 标 Janus TVTA-HFCSQ 及 TVTP-HFCSQ 被成功制备, 其具有明确的化学结构及两面各异官能基团的特点.
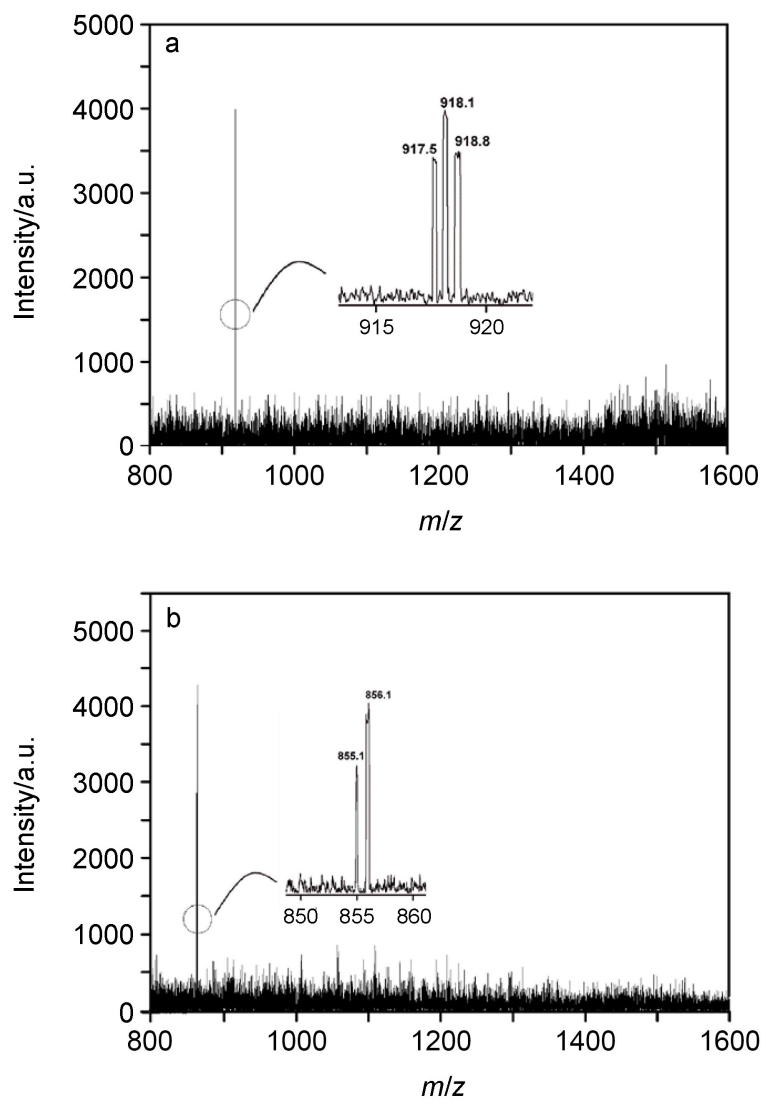

图 8 TVTA-HFCSQ 及 TVTP-HFCSQ 的 Maldi-tof 质谱图 Figure 8 Maldi-tof MS spectra of TVTA-HFCSQ and TVTPHFCSQ

The insert pictures at the corresponding top-right corner were enlarged view of the mass spectrum

\subsubsection{TVTA-HFCSQ 及 TVTP-HFCSQ 结晶性及热稳} 定性能

进一步利用 WAXD (Wide Angle X Ray Diffraction) 对 TVTA-HFCSQ 及 TVTP-HFCSQ 可能存在的结晶结构 与形态进行了表征. 如图 9 所示, 以 TVTA-HFCSQ 为例 分析, 观察到在 $2 \theta=6.72^{\circ} 、 21.10^{\circ} 、 23.63^{\circ}$ 及 $29.82^{\circ}$, 对 应的 $d$ 为 $13.12 、 4.20 、 3.78$ 及 $2.99 \AA$, 有明显的结晶 衍射峰. $2 \theta=6.72^{\circ}$ 处最强的衍射峰被归属为由倍半硅氧 烷笼型结构长程有序的排列引起的. $2 \theta=21.10^{\circ} 、 23.63^{\circ}$ 及 $29.82^{\circ}$ 处是笼型倍半硅氧烷分子菱形结晶的衍射 峰 ${ }^{[21]}$. WAXD 数据表明 TVTA-HFCSQ 及 TVTP-HFCSQ 存在一定的结晶性. 我们进一步希望利用 DSC (Differ- ential Scanning Calorimetry)测试其结晶熔融温度, 发现 其无结晶熔融峰, 这可能是由于 TVTA-HFCSQ TVTPHFCSQ 的结晶不完善, 不呈现结晶熔融峰.

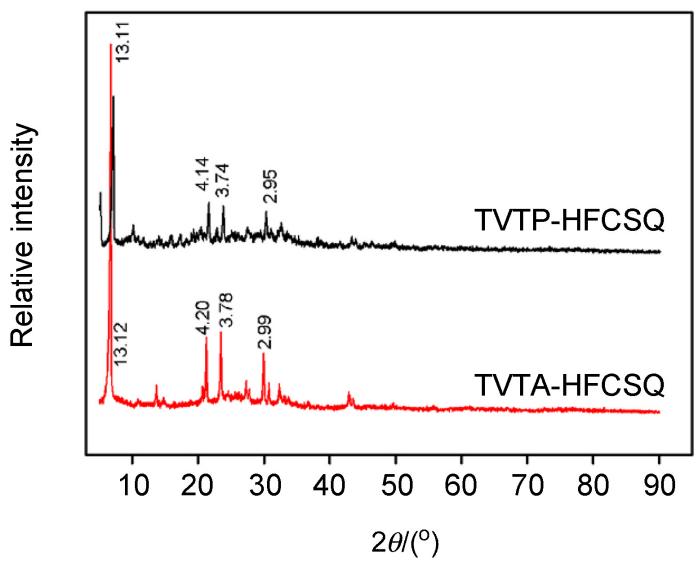

图 9 TVTA-HFCSQ 及 TVTP-HFCSQ 的 WAXD 图 Figure 9 WAXD spectra of TVTA-HFCSQ and TVTP-HFCSQ The peak located at the same position was correspondence to the same crystal face

图 10 为 TVTA-HFCSQ 及 TVTP-HFCSQ 氮气下的 热失重曲线图. 可以看到, 两者的热失重曲线比较相似, 都有两个热分解平台. TVTA-HFCSQ 及 TVTP-HFCSQ 分子中存在几类主要的共价键: $\mathrm{Si}-\mathrm{O} 、 \mathrm{Si}-\mathrm{C} 、 \mathrm{C}-\mathrm{N}$ 及 $\mathrm{C}=\mathrm{C}$ ，其键能分别为 $460 、 347 、 305$ 及 $611 \mathrm{~kJ} / \mathrm{mol}$. 因 此, 热降解应该先由共价键较弱的地方开始. 氮气流下, 两者的热失重曲线在低于 $400{ }^{\circ} \mathrm{C}$ 时都有接近 $20 \%$ 的失 重, 这可以被归结为与乙烯基相关的 $\mathrm{Si}-\mathrm{C}$ 键的断裂, 乙烯基基团的热分解. 对于 TVTA-HFCSQ 及来讲，第 一个热分解平台前 $\mathrm{C}-\mathrm{N}$ 键也会断裂, 氨基会热降解. 进一步提高温度, 苯环开始热降解, 最终残余 $\mathrm{Si}-\mathrm{O}$. TVTA-HFCSQ 及 TVTP-HFCSQ 最终的 $\mathrm{Si}-\mathrm{O}$ 残余率分 别为 57.1 及 $62.0 \mathrm{wt} \%$, 分别高于理论残余率 46.6 及 55.2 $\mathrm{wt} \%$. 这主要是因为笼型倍半硅氧烷分子在降解过程中 残余下来的 $\mathrm{Si}-\mathrm{O}$ 部分在外围形成类似陶瓷防护层, 有 效保护内层快速降解, 并且有可能截留包含一部分苯基 缩合形成的自由碳材料 ${ }^{[22]}$. 结果表明 TVTA-HFCSQ 及 TVTP-HFCSQ 具有优异的耐热稳定性, 无机 $\mathrm{Si}-\mathrm{O}-\mathrm{Si}$ 的刚性内核为其应用于耐高温材料提供了可能. 另外, 如图 11 所示, 在空气中, TVTA-HFCSQ 及 TVTPHFCSQ 热分解行为与其在氮气中总体类似, 存在三个 阶段. 在空气气氛中, 热失重中间过程没有出现明显的 平台期，这与在空气中燃烧更加快速且彻底有关. 并且, TVTP-HFCSQ 及 TVTA-HFCSQ 最终残余率分别为 $52.2 \%$ 及 $43.6 \%$, 这更加接近于理论残余率 $55.2 \%$ 及 $46.6 \%$. 


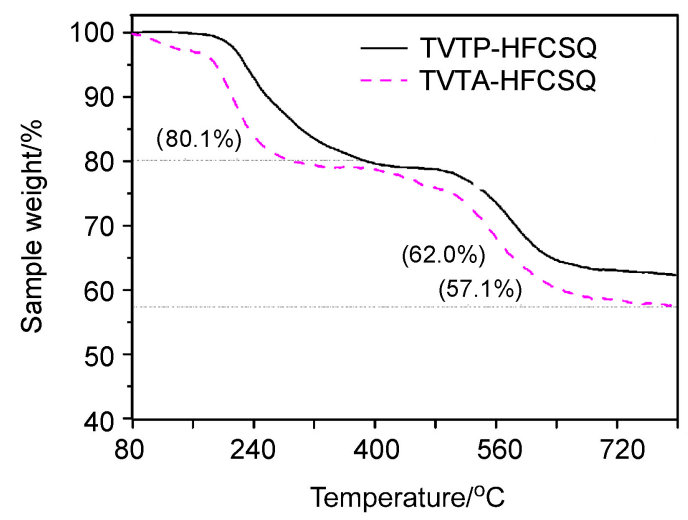

图 10 TVTA-HFCSQ 及 TVTP-HFCSQ 的 TGA 谱图(氮气气 氛)

Figure 10 TGA thermograms of TVTA-HFCSQ and TVTPHFCSQ at a ramp rate of $10{ }^{\circ} \mathrm{C} / \mathrm{min}$ in the $\mathrm{N}_{2}$ flow

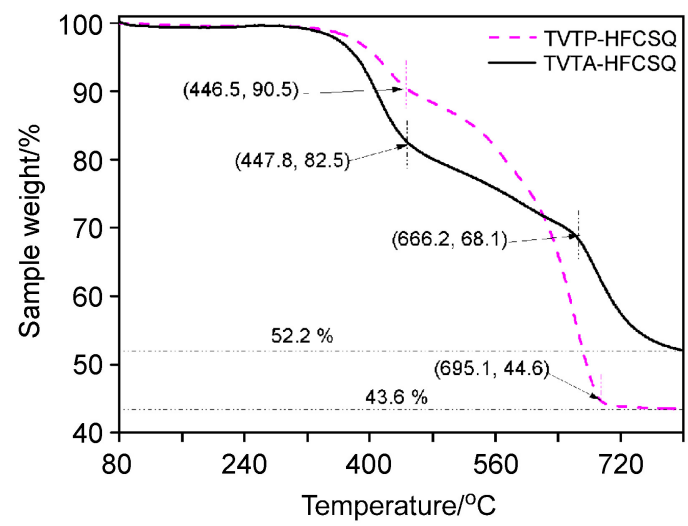

图 11 TVTA-HFCSQ 及 TVTP-HFCSQ 的 TGA 谱图(空气气 氛)

Figure 11 TGA thermograms of TVTA-HFCSQ and TVTPHFCSQ at a ramp rate of $10{ }^{\circ} \mathrm{C} / \mathrm{min}$ in the air flow

1.2.3 TVTA-HFCSQ 及 TVTP-HFCSQ 电子携带及传 输 “超级共轭” 效应

一直以来, 倍半硅氧烷笼子结构被认为是不导电的 介质, 近来, 有文献报道发现其可能存在超级共轭效 应 ${ }^{[23]}$. 我们利用荧光发射及紫外吸收光谱来验证 TVTA-HFCSQ 及 TVTP-HFCSQ 内的大范围电子离域, 侧面也证实了合成的 TVTA-HFCSQ 及 TVTP-HFCSQ 具 有完整的笼型结构. 如图 12 所示, 苯胺、苯、TVTAHFCSQ 及 TVTP-HFCSQ 的最大紫外吸收峰分别位于 286、252、304 及 $263 \mathrm{~nm}$. 比较苯胺与 TVTA-HFCSQ 以及苯与 TVTP-HFCSQ 的最大吸收峰波长, 后者紫外 吸收峰都出现了红移. 同样, 苯胺、苯、TVTA-HFCSQ 及 TVTP-HFCSQ 的荧光发射光谱图也有类似的红移现 象, 由苯胺的苂光发射波长为 $330 \mathrm{~nm}$ 变为 TVTAHFCSQ 的苂光发射波长为 $351 \mathrm{~nm}$; 苯的苂光发射波长 为 $280 \mathrm{~nm}$ 变为 TVTP-HFCSQ 的苂光发射波长为 286 $\mathrm{nm}$. 紫外可见光吸收及荧光发射光谱分析出现的红移 可能是由于 TVTA-HFCSQ 及 TVTP-HFCSQ 的内部共轭 作用引起的, 这也说明倍半硅氧烷笼子存在一定的延伸 电子云共轭的作用, 对于 TVTA-HFCSQ 及 TVTPHFCSQ 来讲, 电子云极有可能从一端经过笼子结构离 域至另一端, 形成更大范围的超级共轭作用, 为 TVTAHFCSQ 及 TVTP-HFCSQ 应用于光电材料或电子屏蔽材 料提供了可能.
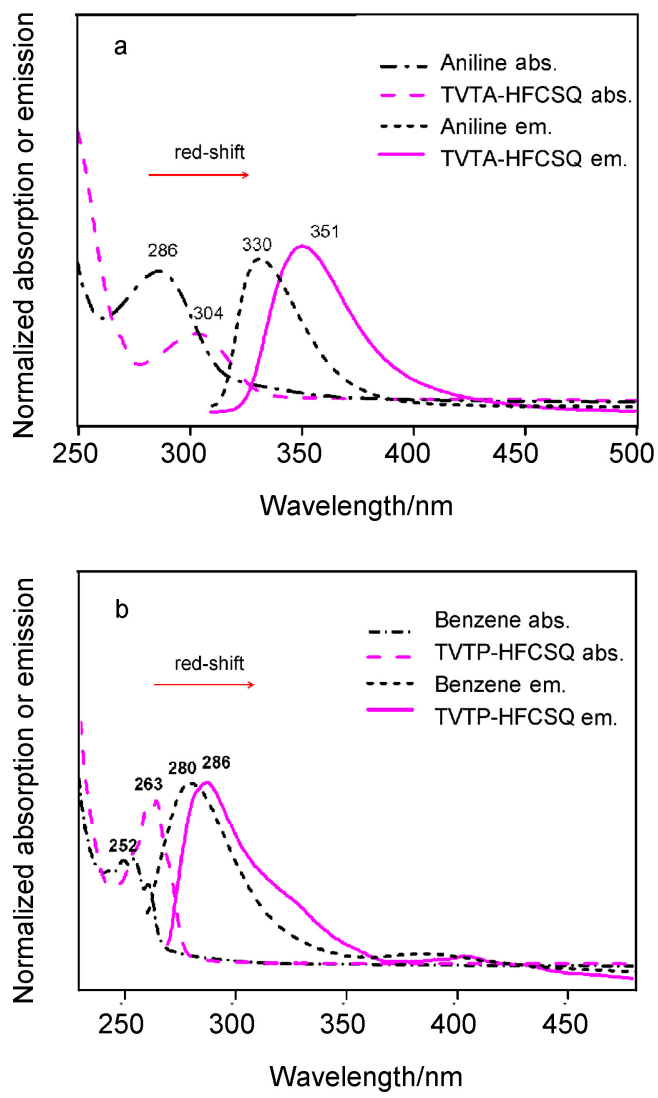

图 12 TVTA-HFCSQ (a)及 TVTP-HFCSQ (b)的 UV 吸收及苂 光发射光谱

Figure 12 UV absorption and PL emission of aniline and TVTA-HFCSQ (a) and benzene and TVTP-HFCSQ (b)

\section{2 结论}

成功探索出一种可宏量制备具有 Janus 结构的异官 能基笼型倍半硅氧烷的新方法, 其包含两步反应: 基于 半笼型倍半硅氧烷模板进行生长以及缩合闭笼. TVTA-HFCSQ 及 TVTP-HFCSQ 数据表征结果完整, 结 构明确，是一类新型的 Janus 功能纳米粒子. 实验同时 证实了倍半硅氧烷笼子结构的电子携带 “超级共轭” 效 应的存在. 另外, 利用其异官的特征, 可以制备出 Janus 各向异性的 CSQ 基聚合物材料, 方便得到更多种类和 功能特性的 CSQ 聚合物. TVTA-HFCSQ 及 TVTP- 
HFCSQ 有可能在主链含 CSQ 聚合物杂化材料、有机光 电材料、太阳能电池以及各向异性材料领域有潜在的应 用. 其准确结构的表征数据完善, 为相关研究人员提供 一定的参考。

\section{3 实验部分}

\section{1 仪器与试剂}

四甲基氢氧化铵五水化合物(TMAH)、氢氧化钠、 邻磺酰苯甲酰亚胺、四丁基氟化铵(TBAF)、三乙胺、茮 基三甲基氢氧化铵(BzTMAH)、苯基三氯硅烷、苯基三 甲氧基硅氧烷、乙烯基三氯硅烷、发烟硝酸、水合肼、 三氯化铁、活性碳粉末, 上海阿拉丁试剂厂, 分析纯; 苯 基笼型倍半硅氧烷(OPS)为实验室制备, 蒸馏水自制. 除有特别说明, 其他材料和试剂使用前均未作处理.

红外光谱(FT-IR)采用美国 Analect 公司 RFX-65A 型 傅里叶红外光谱仪, 固体样品采用溴化钾压片法, 液体 样品溶解在丙酮中然后涂布在溴化钾片上, 红外烘干溶 剂待测. 核磁共振氢谱 $\left({ }^{1} \mathrm{H} N \mathrm{NMR}\right) 、$ 碳谱 $\left({ }^{13} \mathrm{C} N \mathrm{NMR}\right)$ 和硅 谱 $\left({ }^{29} \mathrm{Si} \mathrm{NMR}\right)$ 采用德国-瑞士 Bruker 公司 DRX 400 型 $400 \mathrm{M}$ 超导核磁共振仪, 四甲基硅(TMS)为内标. 基质 辅助激光解吸时间飞行质谱仪 (MALDI-TOF) 使用 AutoflexTM III smartbean 型, 辅助基质为 $\alpha$-氰基-4-羟基 肉桂酸, 为了检测的需要适当加入了钠盐, 分别采用线 性模式和反射模式. 示差扫描量热(DSC)采用 Perkin Elmer 公司 Diamond/Pyris I 型示差扫描量热仪, $\mathrm{N}_{2}$ 气氛, 升温速率 $20{ }^{\circ} \mathrm{C} / \mathrm{min}$, 温度扫描范围为 50 至 $300{ }^{\circ} \mathrm{C}$, 以 DSC 曲线的最大峰值确定物质熔点. 热重分析(TGA)采 用 Perkin-Elmer TGA-6 型热重分析仪, 温度扫描范围为 50 至 $750{ }^{\circ} \mathrm{C}$, 检测合成产物热稳定性. $\mathrm{UV}$ 光谱采用是 UV-8000 型紫外可见光分光光度计, 扫描范围为 800 至 $100 \mathrm{~nm}$. 荧光光谱采用的是 Hitachi F-4600 仪器, 样品 溶解于四氢呋喃, TVTA-HFCSQ、TVTP-HFCSQ 激发波 长分别为 300、 $250 \mathrm{~nm}$; TVTN-HFCSQ 由于硝基的强吸 电子效应, 荧光淬灭. 广角 $\mathrm{X}$ 射线衍射分析(WAXD)采 用是日本 Rigaku D/MAX-1200 型 X 射线衍射仪, 以 $\mathrm{Cu}$ $\mathrm{K} \alpha$ 辐射 $(\lambda=1.54 \AA)$ 为 $\mathrm{X}$ 光源, 经过 $\mathrm{Ni}$ 过滤器, 在室温 下分析测试.

\section{2 实验方法}

3.2.1 七聚苯基开笼型三硅醇 $\left(\mathrm{Ph}-\mathrm{T}_{7}-(\mathrm{OH})_{3}\right)$ 及七聚 苯基开笼型三硅醇钠盐 $\left(\mathrm{Ph}-\mathrm{T}_{7}-(\mathrm{ONa})_{3}\right)$ 的制备

OPS 可控开笼制备 $\mathrm{Ph}_{-} \mathrm{T}_{7}-(\mathrm{OH})_{3}$ 参照文献[13]报道 的方法改进了实验. 典型的投料比为 $n(\mathrm{OPS}): n$ (水) : $n$ (催化剂 $)=1: 1: 2, \mathrm{OPS}$ /异丙醇 $=1 \mathrm{mmol} / 10 \mathrm{~mL}$. 反 应条件为: 氮气保护下磁力搅拌回流反应 $24 \mathrm{~h}$. 采取不 同的催化剂[四甲基氢氧化铵五水化合物(TMAH)、氢氧
化钠、邻磺酰苯甲酰亚胺、四丁基氟化铵(TBAF)、三乙 胺等 $]$ 研究其开笼反应的结果.

苯基三甲氧基硅烷直接水解缩合法制备 $\mathrm{Ph}-\mathrm{T}_{7}-(\mathrm{ONa})_{3}$ 参照文献[14]的方法进行. 典型的配比为: $4.5 \mathrm{~g}$ 苯基三甲氧基硅烷、 $25 \mathrm{~mL}$ 去离子水及 $0.4 \mathrm{~g}$ 氢氧 化钠投入到 $50 \mathrm{~mL}$ 三口烧瓶中, 磁力搅拌下, 回流反应 $5 \mathrm{~h}$, 冷却至室温后, 继续搅拌 $15 \mathrm{~h}$, 停止反应后去除挥 发性组分, 真空干燥箱干燥后得到白色固体产物.

\subsection{2 两类半笼倍半硅氧烷双盖帽的方法}

四乙烯基环硅氧烷四硅醇的合成(TVS)参照文献 [17]. $4.48 \mathrm{~g}$ 乙烯基三甲氧基硅烷、 $0.45 \mathrm{~mL}$ 水、 $3 \mathrm{~mL}$ 乙 醇、 $21 \mathrm{~mL}$ 正己烷、 $1.65 \mathrm{~g}$ 氢氧化钾加入带有回流装置 的 $50 \mathrm{~mL}$ 三口烧瓶中, $20{ }^{\circ} \mathrm{C}$ 反应 $1.5 \mathrm{~h}$, 析出固体产物, 过滤后干燥.

四苯基环硅氧烷四硅醇(TPS)的合成参照文献[17]. $9.9 \mathrm{~g}(50 \mathrm{mmol})$ 苯基三甲氧基硅烷、 $50 \mathrm{~mL}$ 异丙醇为溶 剂、 $0.9 \mathrm{~mL}(50 \mathrm{mmol}) \mathrm{H}_{2} \mathrm{O} 、 2.0 \mathrm{~g}(50 \mathrm{mmol})$ 氢氧化钠, 室 温反应 $12 \mathrm{~h}$. 随着反应的进行, 白色固体产物析出. 反 应结束后，过滤，用适量的异丙醇、甲醇分别洗涤 2 3 次, 产品置于真空干燥箱干燥; 产品溶于四氢呋喃, 加 入醋酸中和, 生成 TPS.

TVS 与 TPS 双盖帽反应参照文献[10]. TVS (2.5 $\mathrm{mmol}) 、 \mathrm{TPS}(2.5 \mathrm{mmol})$ 、苯 $(10 \mathrm{~mL})$ 及 BzTMAH $(0.28$ $\mathrm{mL}, 40 \mathrm{wt} \%$ 甲醇溶液)加入反应瓶, 氮气保护下, 磁力 搅拌室温反应 $12 \mathrm{~h}$, 过滤固体产物, 利用苯、甲醇洗涤 多次, 真空干燥, 得到产物.

\subsection{3四苯基环硅氧烷钠盐(TPSS)的制备}

TPSS 的合成制备参照文献[17]报道的路线合成. 较优的实验典型配比为: $9.9 \mathrm{~g}(50 \mathrm{mmol})$ 苯基三甲氧基 硅烷、 $50 \mathrm{~mL}$ 异丙醇为溶剂、 $0.9 \mathrm{~mL}(50 \mathrm{mmol}) \mathrm{H}_{2} \mathrm{O} 、 2.0$ $\mathrm{g}(50 \mathrm{mmol})$ 氢氧化钠, 室温反应 $12 \mathrm{~h}$. 随着反应的进行, 白色固体产物析出. 反应结束后, 过滤, 用适量的异丙 醇、甲醇分别洗涤 2 3 次, 再溶于适量乙醚中, 加入过 量正己烷中, 析出白色固体产物, 经过真空干燥箱干燥, 得到 $4.8 \mathrm{~g}$ 产物 TPSS, 产率 $60.2 \%$. ${ }^{1} \mathrm{H}$ NMR $(400 \mathrm{MHz}$, DMSO- $\left.d_{6}\right) \delta: 7.79 \sim 7.22(\mathrm{~m}, 301 \mathrm{H}), 7.22 \sim 4.89(\mathrm{~m}$, 114H); IR (KBr) $v: 1578,1448,3369,3220,1119 \quad \mathrm{~cm}^{-1}$; ${ }^{29} \mathrm{Si}$ NMR (400 MHz, DMSO) $\delta:-69.5$.

\subsection{4 四氨基苯基环硅氧烷钠盐(TASS)的制备}

TASS 的合成是通过 TPSS 硝化、还原反应制备的， 参照了苯基笼型倍半硅氧烷硝化反应制备硝基苯基笼 型倍半硅氧烷的实验方法 ${ }^{[24]} .5 .0 \mathrm{~g}$ TPSS 分批次缓慢加 入 $30 \mathrm{~mL}$ 发烟硝酸中(冰水浴反应 $30 \mathrm{~min}$ ), 再移至室温 继续反应 $6 \mathrm{~h}$, 反应结束后利用玻璃漏斗过滤去掉不溶 物, 滴加入 $100 \mathrm{~g}$ 碎冰中, 析出淡黄色固体, 通风梪中过 
夜, 过滤, 去离子水洗、乙醇洗至中性, 真空干燥得到 $5.5 \mathrm{~g}$ 淡黄色固体四硝基苯基硅氧烷钠盐. 参照硝基苯 基笼型倍半硅氧烷经过还原后得到氨基苯基笼型倍半 硅氧烷. 将 $5.0 \mathrm{~g}$ 四硝基苯基硅氧烷钠盐、 $50 \mathrm{mg}$ 三氯化 铁、 $4 \mathrm{~g}$ 活性炭混合加入 $150 \mathrm{~mL}$ 三口烧瓶, 滴加入四氢 呋喃作为溶剂; 反应体系在氮气保护下回流 $15 \mathrm{~min}$, 然 后用恒压漏斗滴加入 $16 \mathrm{~mL} 80 \%$ 水合肼, 混合溶液继续 反应 $6 \mathrm{~h}$ 后, 在布氏漏斗中平铺 $2 \mathrm{~cm}$ 硅藻土, 将反应溶 液倾倒入过滤掉活性炭; 滤液与 $25 \mathrm{~mL}$ 乙酸乙酯合并, 蒸馏水洗, 分层, 干燥, 过滤后浓缩, 浓缩液加入 500 $\mathrm{mL}$ 石油醚中, 析出灰白色固体粉末, 真空干燥; 产品重 新溶解于 $6 \mathrm{~mL}$ 四氢呋喃与 $10 \mathrm{~mL}$ 乙酸乙酯的混合溶液, 滴加入 $500 \mathrm{~mL}$ 石油醚中, 析出产物, 提纯后得到 $3.2 \mathrm{~g}$ TASS, 产率 75.2\%. ${ }^{1} \mathrm{H}$ NMR (400 MHz, DMSO- $\left.d_{6}\right) \delta$ : 6.9 8.1 (m, 2.0H), 5.3 3.7 (d, 1.0H); IR (KBr) v: 1119, $1578,1448,3220,3369 ;{ }^{29} \mathrm{Si}$ NMR (400 MHz, DMSO- $d_{6}$ ) $\delta:-79.1,-71.6$ (两处峰与其氨基在苯环上可能存在 的间位及对位取代有关).

3.2.5 乙烯基三氯硅烷基于 TASS 及 TPSS 生长法制 备相应的四乙烯基二甲氧基衍生物

以 TASS、乙烯基三氯硅烷反应为例说明. $2.50 \mathrm{~g}$ (3.57 mmol)TASS 分散于 $25 \mathrm{~mL}$ 甲醇溶剂, 加入 $100 \mathrm{~mL}$ 圆底烧瓶中; $2.34 \mathrm{~g}$ (14.52 mmol)乙烯基三氯硅烷溶解于 $25 \mathrm{~mL}$ 正己烷中, 通过滴液漏斗加入甲醇溶剂中, 控制 滴加速度, 上述非均相反应体系在氮气保护下室温搅拌 反应 $24 \mathrm{~h}$. 随着反应的进行, 不断有不溶的 $\mathrm{NaCl}$ 析出. 停止反应后, 过滤, 滤液静置分层, 有机层中加入无水 硫酸钠干燥, 去除溶剂后得到 $3.27 \mathrm{~g}$ 淡黄色固体四氨基 苯基四乙烯基二甲氧基衍生物, 产率 $85.1 \%,{ }^{1} \mathrm{H} \mathrm{NMR}$ $\left(400 \mathrm{MHz}\right.$, DMSO- $\left.d_{6}\right) \delta: 7.1 \sim 7.9(\mathrm{~m}, 18.6 \mathrm{H}), 6.1 \sim 5.8$ (m, $14.7 \mathrm{H}), 5.5 \sim 5.2(\mathrm{~s}, 10 \mathrm{H}), 3.2 \sim 3.0(\mathrm{~m}, 25.6 \mathrm{H}) ; \mathrm{IR}$ (KBr) $v: 1119,1382,1578,1448,3352$.

TPSS 与乙烯基三氯硅烷反应与上述实验步骤一致, 得到淡黄色的四苯基四乙烯基二甲氧基衍生物, 产率 $78.4 \%$. ${ }^{1} \mathrm{H}$ NMR (400 MHz, DMSO- $\left.d_{6}\right) \delta: 7.40$ (d, $J=$ $29.0,16.8 \mathrm{H}), 5.96(\mathrm{dd}, J=20.0,11.2 \mathrm{~Hz}, 10 \mathrm{H}), 3.51$ (s, $19.8 \mathrm{H}$ ); IR (KBr) $v: 1118,1382,1579,1448 \mathrm{~cm}^{-1}$.

\subsection{6 闭笼反应制备 TVTA-HFCSQ 和 TVTP-HFCSQ}

TVTA-HFCSQ 的合成: $50 \mathrm{~mL}$ 三口烧瓶中, $1.0 \mathrm{~g}$ 四 氨基苯基四乙烯基二甲氧基衍生物溶于 $20 \mathrm{~mL}$ 甲醇溶 剂, 加入 $15 \mathrm{wt} \% \mathrm{TMAH} / \mathrm{Et}_{3} \mathrm{~N}(\mathrm{~m} / \mathrm{m}=1 / 1)$ 水溶液, 上述 混合体系回流反应 $24 \mathrm{~h}$, 直至生成淡黄色的固体产物析 出. 过滤后用甲醇溶剂洗涤至中性, $40{ }^{\circ} \mathrm{C}$ 真空干燥, 得 到 $0.31 \mathrm{~g}$ 产物, 产率 $37.4 \% .{ }^{1} \mathrm{H} \mathrm{NMR}\left(400 \mathrm{MHz}, \mathrm{CHCl}_{3}\right)$ $\delta: 8.06 \sim 6.75(\mathrm{~m}, 2.1 \mathrm{H}), 6.61 \sim 4.96(\mathrm{~m}, 1.6 \mathrm{H}), 3.36 \sim$
3.68 (s, 1H); IR (KBr) v: 1117, 1108, 1141, 1432, 1610, 1625, 3352; ${ }^{29} \mathrm{Si}$ NMR $\left(400 \mathrm{MHz}, \mathrm{CHCl}_{3}\right) \delta:-77.3$, -82.3 .

TVTP-HFCSQ 的合成步骤如上, 得到的白色结晶 状固体产物有 $0.34 \mathrm{~g}$, 产率 $41.5 \%$. ${ }^{1} \mathrm{H}$ NMR $(400 \mathrm{MHz}$, DMSO- $\left.d_{6}\right) \delta: 8.24 \sim 6.75(\mathrm{~m}, 1.6 \mathrm{H}), 6.52 \sim 5.48(\mathrm{~m}, 1 \mathrm{H})$; IR (KBr) $v: 1116,1108,1142,1432,1612,1625 ;{ }^{29} \mathrm{Si} \mathrm{NMR}$ $\left(400 \mathrm{MHz}, \mathrm{DMSO}-d_{6}\right) \delta:-69.5,-79.2$.

辅助材料 (Supporting Information) 关键中间产物及 产物的核磁共振氢谱谱图和硅谱图谱. 这些材料可以免 费从本刊网站(http://sioc-journal. cn/)上下载

\section{References}

[1] (a) Dickson, W.; Beckett, S.; McClatchey, C.; Murphy, A.; O'Connor, D.; Wurtz, G. A.; Pollard, R.; Zayats, A. V. Adv. Mater. 2015, 27, 5974.

(b) Wang, L.; Li, X.; Zheng, F.; Guo, Y.; Zhang, Z.; Chi, H.; Dong, Y.; Wang, C.; Lu, G. Acta Chim. Sinica 2016, 74, 259 (in Chinese). (王雷, 李雪, 郑斐, 郭玉釒金, 张志强, 迟海军, 董岩, 王翠苹, 卢公昊，化学学报, 2016, 74, 259.)

(c) Luo, M.; Ge, J.; Sun, W.; Zhai, H. Acta Chim. Sinica 2016, 74, 839 (in Chinese).

(罗曼琳, 葛峻羽, 孙文正, 翟慧芳, 化学学报, 2016, 74, 839.)

[2] Lee, A. S. S.; Lee, J. H.; Lee, J.-C.; Hong, S. M.; Hwang, S. S.; Koo, C. M. J. Mater. Chem. 2014, 2, 1277.

[3] (a) Peng, J.; Xing, Y.; Xu, K.; Lin, W.; Wu, J.; Yu, Z.; Zhang, Y.; Chen, M. J. Mater. Chem. C 2015, 3, 2897.

(b) Peng, J.; Lin, W.; Xing, Y.; Xu, K.; Shuxi, G.; Yuanyuan, R.; Chen, M. Mater. Lett. 2015, 143, 1.

[4] Zhou, Z.; Lu, Z.-R. Nanomedicine 2014, 9, 2387.

[5] Xing, Y.; Peng, J.; Xu, K.; Lin, W.; Gao, S.; Ren, Y.; Gui, X.; Liang, S.; Chen, M. Chem.-Eur. J. 2016, 22, 2114.

[6] (a) Cordes, D. B.; Lickiss, P. D.; Rataboul, F. Chem. Rev. 2010, 110, 2081.

(b) Wen, Y.; Liu, A. Chin. J. Org. Chem. 2005, 25, 470 (in Chinese).

(温永向, 刘安华, 有机化学, 2005, 25, 470.)

[7] (a) Kuo, S.-W.; Chang, F.-C. Prog. Polym. Sci. 2011, 36, 1649. (b) Li, D.; Niu, Y.; Yang, Y.; Wang, X.; Yang, F.; Shen, H.; Wu, D. Chem. Commun. 2015, 51, 8296.

[8] Kraus-Ophir, S.; Jerman, I.; Orel, B.; Mandler, D. Soft Matter 2011, 7,8862 .

[9] Jung, J. H.; Laine, R. M. Macromolecules 2011, 44, 7263.

[10] Tateyama, S.; Kakihana, Y.; Kawakami, Y. J. Organomet. Chem. 2010, 695, 898.

[11] Liu, H.; Puchberger, M.; Schubert, U. Chem.-Eur. J. 2011, 17, 5019.

[12] Lin, H.; Wan, X.; Jiang, X.; Wang, Q.; Yin, J. J. Mater. Chem. 2012, 22, 2616.

[13] Li, Z.; Kawakami, Y. Chem. Lett. 2008, 37, 804.

[14] Wang, L.; Li, J.; Li, L.; Zheng, S. J. Polym. Sci., Part A: Polym Chem. 2013, 51, 2079.

[15] Bassindale, A. R.; Liu, Z.; MacKinnon, I. A.; Taylor, P. G.; Yang, Y.; Light, M. E.; Horton, P. N.; Hursthouse, M. B. Dalton. Trans. 2003, 2945.

[16] (a) Sulaiman, S.; Bhaskar, A.; Zhang, J.; Guda, R.; Goodson Iii, T.; 
Laine, R. M. Chem. Mater. 2008, 20, 5563.

(b) Wu, L.; Zang, X.; Yan, S.; Tang, S.; Li, Z. Chin. J. Org. Chem. 2014, 34, 596 (in Chinese).

(吴林, 蔵雄, 阎四海, 唐松青, 李战雄, 有机化学, 2014, 34, 596.)

(c) Zhang, L.; Liu, A.; Zeng, X. Chin. J. Org. Chem. 2007, 27, 424 (in Chinese).

(张利利, 刘安华，曾幸荣，有机化学, 2007, 27, 424.)

[17] (a) Shchegolikhina, O.; Pozdniakova, Y.; Antipin, M.; Katsoulis, D.; Auner, N.; Herrschaft, B. Organometallics 2000, 19, 1077.

(b) Ito, R.; Kakihana, Y.; Kawakami, Y. Chem. Lett. 2009, 38, 364.

[18] Peng, J.; Xu, K.; Cai, H.; Wu, J.; Lin, W.; Yu, Z.; Chen, M. RSC Adv. 2014, 4, 7124.

[19] Bassindale, A. R.; Parker, D. J.; Pourny, M.; Taylor, P. G.; Horton,
P. N.; Hursthouse, M. B. Organometallics 2004, 23, 4400.

[20] Huang, J.-c.; He, C.-b.; Xiao, Y.; Mya, K. Y.; Dai, J.; Siow, Y. P. Polymer 2003, 44, 4491.

[21] Fina, A.; Tabuani, D.; Carniato, F.; Frache, A.; Boccaleri, E.; Camino, G. Thermochim. Acta 2006, 440, 36.

[22] Wang, X.-T.; Yang, Y.-K.; Yang, Z.-F.; Zhou, X.-P.; Liao, Y.-G.; Lv, C.-C.; Chang, F.-C.; Xie, X.-L. J. Therm. Anal. Calorim. 2010, 102, 739.

[23] Xu, K.; Lin, W.; Wu, J.; Peng, J.; Xing, Y.; Gao, S.; Ren, Y.; Chen, M. New J. Chem. 2015, 39, 8405.

[24] (a) Zhang, J.; Xu, R. W.; Yu, D. S. J. Appl. Polym. Sci. 2007, 103, 1004.

(b) Tamaki, R.; Tanaka, Y.; Asuncion, M. Z.; Choi, J.; Laine, R. M. J. Am. Chem. Soc. 2001, 123, 12416.

(Li, L.; Fan, Y.) 